\title{
Preparation, Optimization, and Screening of the Effect of Processing Variables on Agar Nanospheres Loaded with Bupropion HCl by a D-Optimal Design
}

\author{
Jaleh Varshosaz, ${ }^{1}$ Mohammad Reza Zaki, ${ }^{1,2}$ Mohsen Minaiyan, ${ }^{3}$ and Jaafar Banoozadeh ${ }^{2}$ \\ ${ }^{1}$ Department of Pharmaceutics, Novel Drug Delivery Systems Research Centre, School of Pharmacy and Pharmaceutical Science, \\ Isfahan University of Medical Sciences, Isfahan 81746-73461, Iran \\ ${ }^{2}$ Exir Pharmaceutical Company, Borujerd, Iran \\ ${ }^{3}$ Department of Pharmacology, School of Pharmacy and Pharmaceutical Science, Isfahan University of Medical Sciences, \\ Isfahan 81746-73461, Iran
}

Correspondence should be addressed to Jaleh Varshosaz; varshosaz@pharm.mui.ac.ir

Received 28 August 2014; Revised 7 November 2014; Accepted 17 November 2014

Academic Editor: Paola Laurienzo

Copyright (C) 2015 Jaleh Varshosaz et al. This is an open access article distributed under the Creative Commons Attribution License, which permits unrestricted use, distribution, and reproduction in any medium, provided the original work is properly cited.

Bupropion is an atypical antidepressant drug. Fluctuating in its serum levels following oral administration of immediate release dosage forms leads to occasional seizure. The aim of the present work was designing of sustained release bupropion $\mathrm{HCl}$ nanospheres suited for pulmonary delivery. Agar nanospheres were prepared by transferring the w/o emulsion to solid in oil (s/o) suspension. Calcium chloride was used as cross-linking agent and hydroxypropyl $\beta$-cyclodextrin $(\mathrm{HP} \beta \mathrm{CD})$ was used as permeability enhancer. A response surface $\mathrm{D}$-optimal design was used for optimization of nanospheres. Independent factors included in the design were calcium chloride percent, speed of homogenization, agar percent, and HP $\beta$ CD percent. Optimum condition was predicted to be achieved when the calcium chloride was set at 7.19\%, homogenization speed at $8500 \mathrm{rpm}$, agar content at $2 \%$, and $\mathrm{HP} \beta \mathrm{CD}$ at $0.12 \%$. The optimized nanoparticles showed particle size of $587 \mathrm{~nm}$, zeta potential of $-30.9 \mathrm{mV}$, drug loading efficiency of $38.6 \%$, and release efficiency of $51 \%$ until $5 \mathrm{~h}$. The nanospheres showed high degree of bioadhesiveness. D-optimal response surface method is a satisfactory design to optimize the fabrication of bupropion $\mathrm{HCl}$ loaded agar nanospheres and these nanospheres can be successively exploited to deliver bupropion in a controlled manner for a sufficiently extended period.

\section{Introduction}

Bupropion is an atypical short-acting aminoketone antidepressants drug $[1,2]$ which inhibits the reuptake of dopamine and norepinephrine [3]. It was patented for the first time in 1974 [4] and released onto the world market in 1985 but was briefly withdrawn due to seizures incidence. It was reintroduced in 1989 after the daily recommended dose was reduced to lower seizure likelihood [3].

To address the dose-related risk of seizures associated with high peak concentration of the drug following oral administration, bupropion hydrochloride is administered in divided doses or as sustained release dosage forms $[1,2,5,6]$.

Bupropion has numerous therapeutic indications including depression [7], smoking cessation [8], sexual dysfunction [9], obesity [10], attention deficit hyperactivity disorders
[11], and seasonal affective disorders [12]. Following oral administration of immediate release forms of bupropion, peak plasma concentration is usually achieved within 2 hours. The half-life of the postdistributional phase of bupropion ranges from 8 to 24 hours. Bupropion has also a relatively high volume of distribution of $18.6 \mathrm{~L} / \mathrm{kg}[2,13]$.

Following absorption from gastrointestinal tract, bupropion undergoes extensive first-pass metabolism with oral bioavailability of only $5 \%$ resulting in formation of metabolites which are less potent than the parent molecule while being more convulsion inducing $[14,15]$.

To overcome the shortcomings of currently available dosage forms, that is, (i) fluctuation in plasma level due to immediate release forms and (ii) conversion of parent molecule to its metabolites through first-pass effect, it is worth it to try other routes of administration in context of 
sustained release of the drug more common form of which being nanoparticles.

Among various natural polymers available, agar and agarose (fractions of agar) have been used to prepare microand nanoparticles by several workers [17-21]. Agarose hydrogel as nanoparticulate drug delivery system has the advantage of being administrable via different parenteral ways such as subcutaneous, intramuscular, intravenous, and pulmonary routes [17].

Agar is composed of two polysaccharides, agarose, and agaropectin (Figure 1). Agarose contains 1, 3 linked D-galactose and 1, 4 linked 3, 6 anhydro L-galactose units, with very few hydroxyls being sulphated, useful as a material for the gel formation. Agaropectin is a more complex structure than agarose, containing in addition to $D$-galactose and 3, 6 anhydro galactose units, $D$-gluconic acid, pyruvic acid, and a much higher proportion of sulphate ester groups [20].

Agar shows some interesting physical properties. It is not soluble in cold water but is soluble in boiling water. Its solution forms thermally reversible hydrogels while being cooled down below its gelation temperature $\left(31-36^{\circ} \mathrm{C}\right)$. The reversible gel-to-sol transition for the agarose hydrogel does not occur below the melting point $\left(65-85^{\circ} \mathrm{C}\right)[22]$.

In production of nanoparticles, different techniques have been used based on creating w/o emulsion, the aqueous phase of which consists of polymer solution dispersed in an oily continuous phase which is corn oil [17], soybean oil [18], liquid paraffin [20, 23], or methylene chloride [19]. Some researchers have tried to cover the agar microparticles simultaneously with a layer of PLGA making use of a phase separation technique [19].

Some reported methods for manufacturing of agar particles mentioned in literature are emulsification [24, 25], spraying-gelation [26], membrane emulsification [13], and nanoprecipitation [21].

Mucoadhesion is the other feature of agar which makes it an appropriate candidate for use as the matrix of nanoparticles [20].

Fabrication of nanoparticles loaded with various therapeutically active molecules is currently under extensive research by scientists worldwide. Nanocarriers used in pulmonary delivery of systemic and local drugs are mainly made of natural or synthetic polymers. Agar, a natural polymer, has not received the appropriate attention it deserves by the researchers. A limited number of works are published in the literature making use of agar or agarose to deliver drugs in the form of nano- or microparticles, none of them related to the respiratory tract. Wang and $\mathrm{Wu}[19]$ entrapped protein molecules in agarose which was further encapsulated within poly(lactic-co-glycolic acid) (PLGA) microspheres. These potentially could be administered in form of different dosage forms via various delivery routes. In another study, the same authors prepared agar nanoparticles, employing ovalbumin as a model drug [17]. These nanoparticles were declared as a possible vehicle for sustained delivery of peptides and proteins. Ju et al. [18] prepared agar nanoparticles containing protein molecules to be delivered from a suitable route. Manjunatha et al. [23] prepared sustained release diclofenac containing agar beads for application in delivery of the

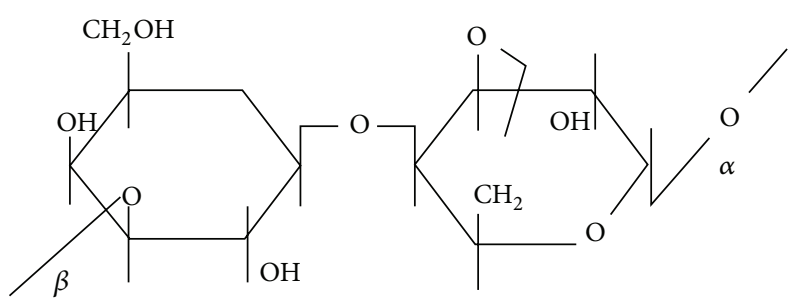

Agarose

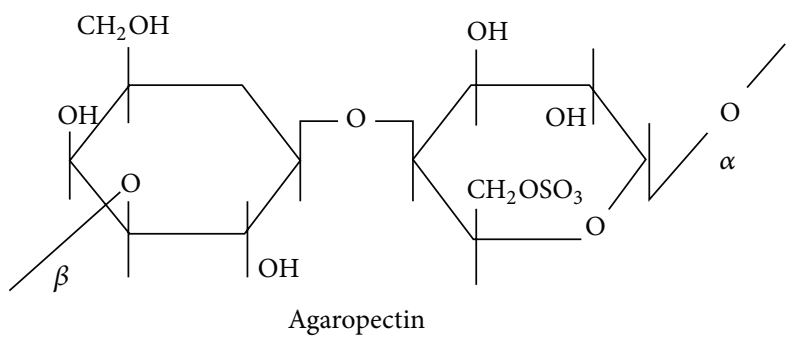

FIGURE 1: Chemical structure of subunits of agar including agarose and agaropectin.

drug. Linghui et al. [27] proposed precipitation method for fabricating nano-agar particles. Sustained release of mucoadhesive agar microspheres loaded with metformin $\mathrm{HCl}$ for oral delivery of metformin was tried by Bera et al. [20].

On the other hand, pulmonary delivery of various therapeutic molecules in context of polymers other than agar is being attempted currently by several workers, but pulmonary delivery of drugs using agar nanocarrier has not been reported so far to the best of our knowledge. So considering the unique features of agar and shortcomings of oral delivery of bupropion $\mathrm{HCl}$ mentioned through the previous paragraphs, it is worth trying out to administer bupropion $\mathrm{HCl}$ via pulmonary route in the form of drug loaded agar nanospheres.

The main drawback of all reported methods for preparation of agar nanoparticles is their severe stickiness which prevents their wide use. In the present study, we have tried to design nanoparticles of bupropion for pulmonary administration by incorporating it into agar nanoparticles adopting the above mentioned general method with some modifications to prevent their stickiness and obtaining freely reconstituting sediments. Calcium chloride which bears a relative negative charge was used for cross-linking of the agar nanoparticles to retard the release rate of bupropion hydrochloride. As these nanoparticles are intended to be administered via pulmonary route, hydroxypropyl beta cyclodextrin (HP $\beta C D$ ) which shows the lowest cell toxicity on pulmonary epithelial cell lines and is the only modified $\beta C D$ cited in the FDA list of Inactive Pharmaceutical Ingredients [28] was also used as a penetration enhancer.

\section{Materials and Methods}

2.1. Materials. Agar was obtained from Narico (Germany), liquid paraffin was obtained from Golnoosh Company (Iran), hydroxypropyl beta cyclodextrin which is not a hazardous 
or not classified as dangerous substance was purchased from Sigma (US), Bupropion $\mathrm{HCl}$ (99.8\% pure) was purchased from Dipharma (Italy), and calcium chloride and methanol HPLC grade were purchased from Merck Chemical Company (Germany). All other reagents were of analytical grades.

2.2. Preparation of Nanospheres. Agar (100, 150, and $200 \mathrm{mg})$ was added to $10 \mathrm{~mL}$ of water. The resultant suspension was heated up to boiling temperature to dissolve the agar and then cooled down to $45^{\circ} \mathrm{C}$ keeping the vessel covered meanwhile to prevent the loss of water. Calcium chloride, $\mathrm{HP} \beta \mathrm{CD}$, and bupropion $\mathrm{HCl}$ previously dissolved in appropriate amounts of water (according to Table 1 ) were added at this temperature and mixed for three minutes. The resultant solution was added under homogenization to $40 \mathrm{~mL}$ of liquid paraffin previously warmed up to $40^{\circ} \mathrm{C}$. After homogenizing at predetermined speeds by homogenizer (Ika T25 basic, Janken and Kunkel GmbH, Germany) for 2 minutes, the suspension was cooled down below $20^{\circ} \mathrm{C}$ by immersing the vessel in ice water bath. Centrifugation at $8000 \mathrm{rpm}$ for 5 minutes was performed to settle the nanospheres. The sediment was washed three times by redispersing in $5 \mathrm{~mL}$ of methylene chloride. The final sediment was dispersed in $3 \mathrm{~mL}$ of ethanol, added to $3 \mathrm{~g}$ of mannitol, mixed thoroughly to get a homogenized paste, and left overnight to get air dried.

2.3. Morphological Study of Nanospheres. The scanning electron microscopy (SEM) studies were conducted on a Philips XL 30 instrument (USA) operating at $26 \mathrm{keV}$.

\subsection{Zeta Potential, Particle Size, and Size Distribution Mea-} surement. Particle size and zeta potential measurements were performed by Malvern ZetaSizer (Model 3000 NS, UK).

2.5. Drug Loading Efficiency Determination. Accurately weighted $40 \mathrm{mg}$ of nanoparticles was dispersed in $20 \mathrm{~mL}$ of phosphate buffer ( $\mathrm{pH} 7.2)$, shaken (100 rpm) for 24 hours, and assayed after filtration through $0.22 \mu \mathrm{m}$ syringe filter by HPLC method according to Loboz et al. [29] method. The analysis was performed by a Knauer HPLC system with Chromgate Software version 3.1 equipped with a binary pump, Smartline-1000-1 and Smartline-1000-2, and a UV detector (Smartline-UV-2500, variable wavelength, programmable, Berlin, Germany), an online solvent vacuum degasser, and a manual sample injector. Analysis was carried out on a C18 column $(25 \mathrm{~cm} \times 4.6 \mathrm{~mm}$, particle size of $5 \mu \mathrm{m}$ ) from Agilent (USA). The mobile phase consisted of methanol and $0.05 \mathrm{M}$ phosphate buffer adjusted to $\mathrm{pH} 5.5$ with phosphoric acid (85\%) before addition of methanol $(45: 55 \mathrm{v} / \mathrm{v})$. The flow rate was maintained at $1.0 \mathrm{~mL} / \mathrm{min}$ at ambient temperature.

Loading efficiency (LE) was calculated according to the following equation:

$$
\begin{aligned}
\mathrm{LE} \%= & \left(\frac{\text { Analyzed weight of drug in nanoparticles }}{\text { Theoretical weight of drug loaded in the system }}\right) \\
& \times 100 .
\end{aligned}
$$

2.6. In Vitro Release Study. Nanospheres equivalent to $2.5 \mathrm{mg}$ of bupropion $\mathrm{HCl}$ were dispersed in $3 \mathrm{~mL}$ of phosphate buffer ( $\mathrm{pH} 7.4$ ), decanted in dialysis bag, and placed in $200 \mathrm{~mL}$ of $37^{\circ} \mathrm{C}$ buffer. At predetermined time intervals, $3 \mathrm{~mL}$ of release medium was withdrawn and analyzed spectrophotometrically at $298 \mathrm{~nm}$ to determine the released amount of bupropion $\mathrm{HCl}$. The withdrawn samples were replaced by $3 \mathrm{~mL}$ of fresh $37^{\circ} \mathrm{C}$ buffer.

Release efficiency (RE) at 300 minutes was calculated according to the following equation for each formulation:

$$
\mathrm{RE}_{300} \%=\frac{\int_{0}^{300} y d t}{y_{100} \times t} \times 100 \text {, }
$$

where $y$ is the released percent at time $t$.

2.7. Optimization Method. D-optimal design minimizes the determinant of the $\left(X^{\prime} X\right)^{-1}$ matrix. They are built algorithmically to provide the most accurate estimates of the model coefficients. In this study, Design-Expert Software (version 7.0.0, Stat-Easc, Inc., Minneapolis, MN, USA) was used to develop a D-optimal response surface methodology (RSM) design based on the independent factors of calcium chloride percent $\left(X_{1}\right.$ or $\left.\mathrm{C}\right)$, homogenization speed $\left(X_{2}\right.$ or $\left.\mathrm{S}\right)$, agar percent $\left(X_{3}\right.$ or $\left.\mathrm{A}\right)$, and $\mathrm{HP} \beta \mathrm{CD}$ percent $\left(X_{4}\right.$ or $\left.\mathrm{H}\right)$. Based on the D-optimal design, a total of 25 experiments were performed. This allowed the choice of the best model from the linear model (3a), a two-factor interaction model (3b), and a quadratic model (3c) based on the $F$-value derived from ANOVA, and the $R^{2}$, predicted $R^{2}$, and adjusted $R^{2}$.Consider

$$
\begin{gathered}
Y_{i}=\beta_{0}+\beta_{1} X_{1}+\beta_{2} X_{2}+\beta_{3} X_{3}+\beta_{4} X_{4} \\
Y_{i}=\beta_{0}+\beta_{1} X_{1}+\beta_{2} X_{2}+\beta_{3} X_{3}+\beta_{4} X_{4} \\
+\beta_{12} X_{1} X_{2}+\beta_{13} X_{1} X_{3}+\beta_{14} X_{1} X_{4} \\
+\beta_{23} X_{2} X_{3}+\beta_{24} X_{2} X_{4}+\beta_{34} X_{3} X_{4} \\
Y_{i}=\beta_{0}+\beta_{1} X_{1}+\beta_{2} X_{2}+\beta_{3} X_{3}+\beta_{11} X_{1}^{2} \\
+\beta_{22} X_{2}^{2}+\beta_{33} X_{3}^{2}+\beta_{44} X_{4}^{2}+\beta_{12} X_{1} X_{2}+\beta_{13} X_{1} X_{3} \\
+\beta_{14} X_{1} X_{4}+\beta_{23} X_{2} X_{3}+\beta_{24} X_{2} X_{4}+\beta_{34} X_{3} X_{4}
\end{gathered}
$$

In these equations, $Y_{i}$ is the predicted value on any of the chosen measured responses (i.e., $Y_{1}$ : particle size, $Y_{2}$ : zeta potential, $Y_{3}$ : drug payload efficiency, and $Y_{4}$ : percentage of release efficiency after 5 hours), $\beta_{0}$ is an intercept, $\beta_{1}, \beta_{2}, \beta_{3}$, and $\beta_{4}$ are linear coefficients, $\beta_{12}, \beta_{13}, \beta_{14}, \beta_{23}, \beta_{24}$, and $\beta_{34}$ are the coefficients of the two-way interaction terms, and $X_{1}$, $X_{2}, X_{3}$, and $X_{4}$ are the independent experimental variables which were selected based on the results from a preliminary study. Design-Expert version 7 (Stat-Ease, USA) was used for statistical analysis of the data. The Pvalues less than 0.05 were considered to be statistically significant. In cases of getting insignificance models $(P>0.05)$, model reduction was performed to get a significant one.

For each response, the model suggested by the software was used to fit the data and the mathematical equation 


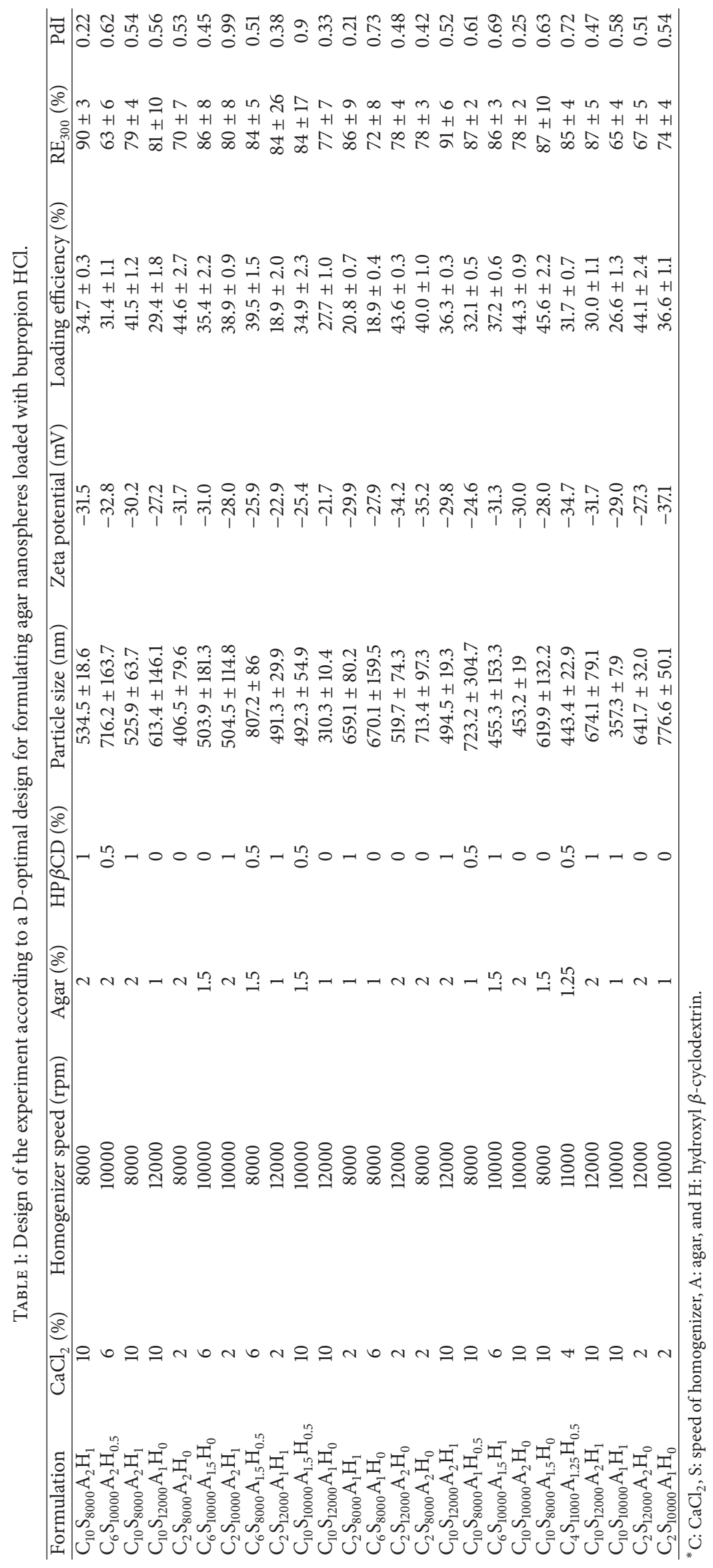


suggested by the software was solved to get the optimal points. A two-factor model was used to assess the relationship between the studied variables with the particle size and zeta potential while, a quadratic model for release efficiency and a linear model for loading efficiency. In cases of getting insignificance models $(P>0.05)$, model reduction was performed to get a significant one.

Optimization of response factors was performed for minimizing the particle size while maximizing the absolute value of zeta potential, loading efficiency, and $\mathrm{RE}_{300}$. Solution provided by the software with the greatest desirability was chosen as the optimum condition and, after executing the experiment based on the suggested values for the independent factors, the real responses were compared with the predicted ones and error percentages were calculated to evaluate the predictive ability of the models.

Design expert software produced 25 runs; the composition of each run is given in Table 1.

2.8. Mucoadhesion Evaluation. The extent of mucoadhesion was measured according to Varshosaz and Dehghan [30] method with some modification. Briefly, the calibration curve was produced by measurement of the absorbance of different concentrations of mucin in acetate buffer $(\mathrm{pH} 4.5)$ at $\lambda_{\max }=$ $500 \mathrm{~nm}$. Nanoparticles were added to acetate buffer ( $\mathrm{pH} 4.5$ ) containing mucin $(1 \mathrm{mg} / \mathrm{mL})$ and were mixed for 1 hour. After centrifugation, the absorbance of the supernatant was determined at the same $\lambda_{\max }$. The concentration of free mucin was calculated from the calibration curve. The percent of mucin adsorbed on the surface of nanospheres which is an indicator of the extent of mucoadhesion was calculated according to the following equation:

$$
A=\left(1-C_{\text {free }}\right) \times 100 \text {, }
$$

where $A$ is the percent of adsorbed mucin and $C_{\text {free }}$ is the concentration of free mucin in the supernatant in $\mathrm{mg} / \mathrm{mL}$.

The optimized formulation was prepared with various concentrations of hydroxypropyl $\beta$-cyclodextrin $(0,0.5$ and $1 \%)$ and the mucoadhesion of the optimized nanospheres was measured in acetate buffer. Also the mucoadhesion of the optimized formulation was checked in the simulated lung fluid (Gamble's solution) [16] which its composition is mentioned in the following paragraph.

2.9. Stability Test of Nanoparticles. Considering that bupropion release percentage from the optimized nanospheres after 5 hours was about $50 \%$, the release data were fitted with different kinetic models including the first order release kinetic $\left[M=Q_{0}\left(1-e^{-K t}\right)\right]$, the zero order $\left(Q=Q_{0}+K t\right)$, and Higuchi model $\left(Q=K t^{1 / 2}\right)$. In these equations, $M$ is the amount of drug remaining to be released in time $t, Q$ is the amount of drug released at time $t, Q_{0}$ is the amount of initially loaded drug, and $K$ is the drug release constant. The best model which was better fitted to the release data was chosen according to the highest regression coefficient and the time required for the total release of the drug was anticipated according to that model. The $r^{2}$ values for these models were 0.9411, 0.8913, and 0.9439, respectively. Therefore, the Higuchi model was chosen and the time required for total release of the drug was calculated to be about 9 hours (the Higuchi model equation was $y=5.3491 x-5.8186$, with $r^{2}=0.9439$ ). Therefore, the stability of the optimized nanospheres in simulated lung fluid (Gamble's solution) [16] regarding the particle size, zeta potential, and drug loading percent was studied after 9 hours which was a time period almost necessary for releasing of the total loaded drug from the nanospheres. The composition of the Gamble's solution included magnesium chloride $0.095 \mathrm{~g} / \mathrm{lit}$, sodium chloride $6.019 \mathrm{~g} / \mathrm{lit}$, potassium chloride $0.298 \mathrm{~g} / \mathrm{lit}$, disodium hydrogen phosphate $\left(\mathrm{Na}_{2} \mathrm{HPO}_{4}\right) \quad 0.126 \mathrm{~g} / \mathrm{lit}$, sodium sulfate $0.063 \mathrm{~g} / \mathrm{lit}$, calcium chloride dehydrate $0.368 \mathrm{~g} / \mathrm{lit}$, sodium acetate $0.574 \mathrm{~g} / \mathrm{lit}$, sodium hydrogen carbonate $\left(\mathrm{NaHCO}_{3}\right) 2.604 \mathrm{~g} / \mathrm{lit}$, sodium citrate dihydrate $0.097 \mathrm{~g} / \mathrm{lit}$, and $\mathrm{pH} 7.4$ [16].

\section{Results and Discussion}

3.1. Preparation of Agar Nanoparticles. The exceptional property of agar solution, that is, gel/sol transition at about $85^{\circ} \mathrm{C}$ and backward sol/gel transition at $35-40^{\circ} \mathrm{C}$, makes the basis of its use in formation of micro- and nanoparticles. According to Maa and Hsu [31] and Tacholakova et al. [32], during emulsification by homogenization, equilibrium state is reached within 3 to 4 minutes of homogenization. With respect to preliminary experiments, two minutes of homogenization was decided to provide a satisfactory load while reducing the size down to nanofield.

Since these particles are formed out of a primary w/o emulsion, they are expected to have spherical shape. The presence of some agents, for example, cross-linkers in the formulation system, may somehow deviate the shape from a complete sphere.

As agar particles are very sticky, harvesting the particles and separating them in large amounts is a challenging task. They are not dissociated easily upon further reconstitution and this has compelled the scientist to obtain them in very low concentrations $[17,18]$. To the best of our knowledge, nothing is mentioned in the literature on the final separation of the particles in large amounts so far. We have tried a simple technique consisting of adsorption of the final particles on mannitol powder. Washing the particles with ethanol helps to more rapid drying of the particles and inhibits their aggregation to a considerable extent.

3.2. Particle Size, Size Distribution, and Zeta Potential. The results of measuring of particle size, polydispersity index, and zeta potential of different formulations are seen in Table 1 . The particle size of nanospheres obtained in different runs of the experiment was in the range of $310 \mathrm{~nm}$ (for $\mathrm{C}_{10} \mathrm{~S}_{12000} \mathrm{~A}_{1} \mathrm{H}_{0}$ ) to $807 \mathrm{~nm}$ (for $\mathrm{C}_{6} \mathrm{~S}_{8000} \mathrm{~A}_{1.5} \mathrm{H}_{0.5}$ ).

The obtained data were analyzed by the Design Expert Software according to the criteria mentioned in Section 2.7 and the optimum conditions for production of the nanospheres were suggested by the software. Table 2 shows the results of regression analysis for particle size, zeta potential, drug release, and drug loading efficiency fitted to the two-factor model (in the two former cases), quadratic and linear equations, respectively. As seen in Table 2, the 
TABLE 2: Regression analysis for particle size, zeta potential, drug loading efficiency, and $\mathrm{RE}_{300} \%$.

\begin{tabular}{|c|c|c|c|c|c|c|c|c|c|c|c|c|}
\hline \multirow{3}{*}{ Factor } & \multicolumn{9}{|c|}{ Responses } & \multirow{2}{*}{\multicolumn{3}{|c|}{$\mathrm{RE}_{300} \%$}} \\
\hline & \multicolumn{3}{|c|}{ Particle size } & \multicolumn{3}{|c|}{ Zeta potential } & \multicolumn{3}{|c|}{ Drug loading efficiency \% } & & & \\
\hline & C.E & Std. error & $P$ value & C.E & Std. error & $P$ value & C.E & Std. error & $P$ value & C.E & Std. error & $P$ value \\
\hline Int & +561.70 & 24.58 & 0.1004 & -29.37 & 0.64 & 0.0118 & +33.70 & 1.03 & $<0.0001$ & +81.31 & 3.06 & 0.0289 \\
\hline$X_{1}$ & - & - & - & +1.27 & 0.73 & 0.0969 & - & - & - & +0.84 & 1.48 & 0.5760 \\
\hline$X_{2}$ & -60.02 & 30.53 & 0.0626 & - & - & - & - & - & - & +0.73 & 1.59 & 0.6516 \\
\hline$X_{3}$ & -3.45 & 27.94 & 0.9030 & - & - & - & +6.31 & 1.18 & $<0.0001$ & -0.28 & 1.43 & 0.8456 \\
\hline$X_{4}$ & - & - & - & +0.34 & 0.72 & 0.6386 & -3.21 & 1.15 & 0.0106 & - & - & - \\
\hline$X_{1}{ }^{2}$ & - & - & - & - & - & - & - & - & - & 5.29 & 3.70 & 0.1708 \\
\hline$X_{2}{ }^{2}$ & - & - & - & - & - & - & - & - & - & 5.88 & 2.88 & 0.0565 \\
\hline$X_{3}{ }^{2}$ & - & - & - & - & - & - & - & - & - & -11.87 & 3.70 & 0.0052 \\
\hline$X_{1} X_{3}$ & - & - & - & - & - & - & - & - & - & 3.87 & 1.52 & 0.0211 \\
\hline$X_{1} X_{4}$ & - & - & - & -2.41 & 0.78 & 0.0053 & - & - & - & - & - & - \\
\hline$X_{2} X_{3}$ & +66.11 & 32.73 & 0.0563 & - & - & - & - & - & - & - & - & - \\
\hline \multicolumn{13}{|c|}{ Lack of fit values } \\
\hline$F$ value & & 0.52 & & & 1.05 & & & 2.78 & & & 1.18 & \\
\hline$P$ value & & 0.8524 & & & 0.5270 & & & 0.1315 & & & 0.4595 & \\
\hline
\end{tabular}

$X_{1}: \mathrm{CaCl}_{2}$ percent, $X_{2}$ : homogenization speed, $X_{3}$ : agar percent, $X_{4}$ : HP $\beta C D$ percent, C.E: coefficient estimate in terms of actual factors, and Int: intercept; the positive sign of the factor represents a synergistic effect on the response, while a negative sign means an antagonist relationship.

TABLE 3: Summary of results of regression analysis for responses $Y_{1}, Y_{2}, Y_{3}$, and $Y_{4}$.

\begin{tabular}{lccccc}
\hline Model & $R^{2}$ & Adjusted $R^{2}$ & Predicted $R^{2}$ & Mean & S.D. \\
\hline$Y_{1}$ (particle size) & 0.2522 & 0.1454 & -0.0439 & 564.30 & 121.77 \\
$Y_{2}$ (Zeta potential) & 0.4004 & 0.3147 & 0.1353 & -29.56 & 3.14 \\
$Y_{3}$ (drug loading efficiency) & 0.6135 & 0.5783 & 0.5034 & 34.59 & 10.63 \\
$Y_{4}\left(\mathrm{RE}_{300} \%\right)$ & 0.5559 & 0.3731 & 0.0260 & 79.96 & 6.11 \\
\hline
\end{tabular}

insignificant lack of fit $(P>0.05)$ shows the suitability of the fitted models. Positive sign $(+)$ represents a synergistic effect on the response, while a negative sign (-) means an antagonist relationship. Phrases composed of two factors indicate the interaction terms and phrases with second-order factors stand for the nonlinear relationship between the response and the variable.

As Table 2 indicates, all studied models fitted to data were significant $(P<0.05)$ except for particle size of nanospheres $(P>0.05)$ and, among the predicted responses, all coefficients for loading efficiency were significant $(P<$ 0.05 ). Table 2 also shows that the homogenization speed and agar content had a decreasing effect on the particle size while their interactions had an increasing effect on this response. Nevertheless, all these effects were insignificant $(P>0.05)$. $\mathrm{CaCl}_{2}$ concentration and $\mathrm{HP} \beta \mathrm{CD}$ increased the zeta potential with a nonsignificant effect $(P>0.05)$, although their interaction reduced it significantly $(P<0.05)$.

The multiple correlation coefficients $\left(R^{2}\right)$ of the model for particle size, zeta potential, drug loading, and $\mathrm{RE}_{300} \%$ explained by the model are shown in Table 3. Results show that the predicted $R^{2}$ is in reasonable agreement with the adjusted $R^{2}$, indicating the adequacy of the model to predict the responses.

Figure 2 shows the effect of different studied variables on the particle of the nanospheres. Though the model relating the studied particle size to the input factors was not significant for any of the variables, there was a reduction in particle size with the increasing speed of homogenization (Figure 2) which was also reported in previous works [31]. According to Narsimhan and Goel $[33,34]$, the size of droplets in the dispersed phase during emulsification by homogenizer relates to counteraction of two opposite processes: breaking down of the droplets to smaller ones and coalescence of smaller droplets to form larger ones. The final size, that is, the ultimate equilibrium emulsion droplet size, depends upon the equilibration point between these two oppositely directed events.

The weak correlation $(P=0.0626)$ of particle size with speed of homogenization (Figure 2) in this study can be explained with the fact that the whole range of applied speed in this study, that is, $8000 \mathrm{rpm}$ to $12000 \mathrm{rpm}$, was probably around the ultimate equilibrium emulsion droplet size.

Slight decrease in particle size or negative effect of $\mathrm{CaCl}_{2}$, as the cross-linking agent of agar, on the particle size of nanospheres (Table 2, Figure 2) can be due to partial center directed contraction that is exerted upon the spheres by the cross-linker.

Increasing the amounts of $\mathrm{HP} \beta \mathrm{CD}$ led to some insignificant decrease in particle size (Figure 2) which can be due to the amphoteric structure of $\mathrm{HP} \beta \mathrm{CD}$, bestowing some surface acting property on it and finally reducing the droplet size of the primary emulsion. 


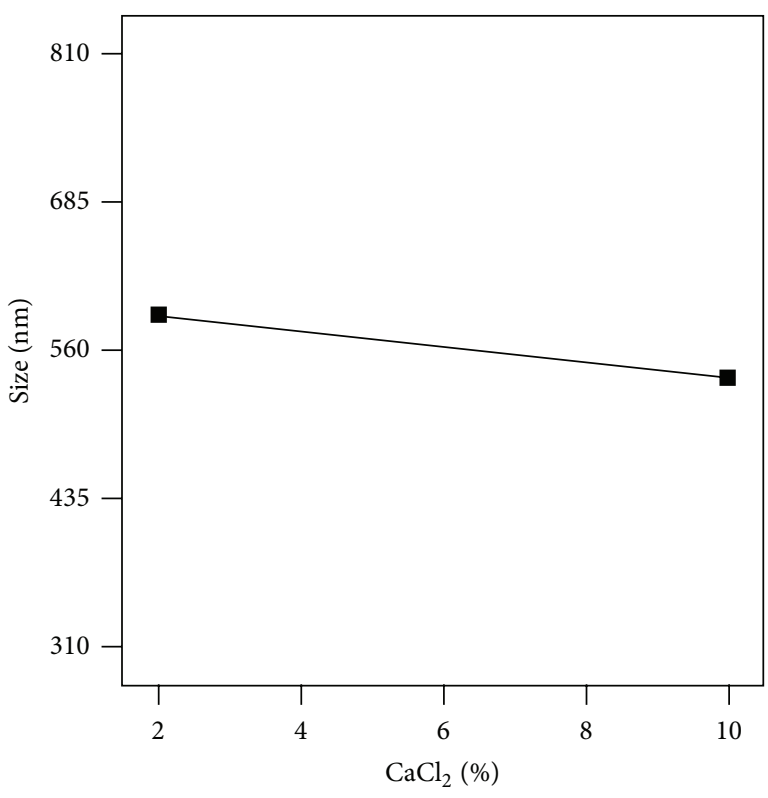

(a)

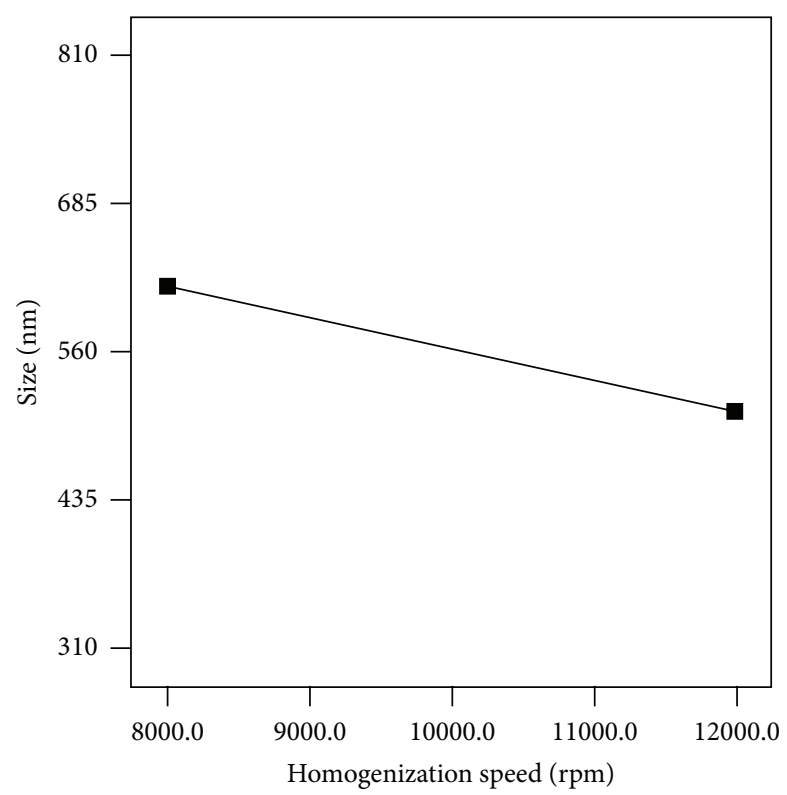

(b)

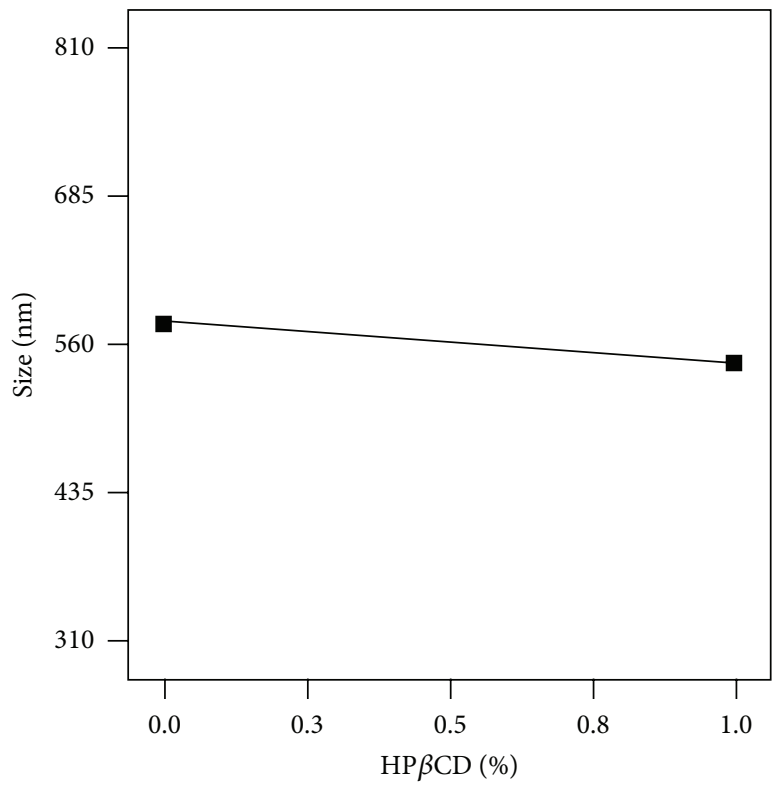

(c)

FIgURE 2: Correlation between particle size of agar nanospheres and (a) $\mathrm{CaCl}_{2} \%$, (b) homogenizer speed, and (c) $\mathrm{HP} \beta \mathrm{CD} \%$.

As can be expected, change in agar contents of the emulsion had no effects on droplet size (Figure 2).

Cross-linking of the agar with calcium ions partially neutralizes the negative charge of the agar molecules and subsequently diminishes the absolute measure of zeta potential (Figure 3). Increasing amounts of $\mathrm{HP} \beta \mathrm{CD}$ by covering more and more surface of the nanospheres through interaction with nonpolar projected groups can decrease the surface polar density which is finally translated into the decreasing of absolute amounts of zeta potential (Figure 3).

3.3. Morphology of Nanospheres. As could be seen in Figure 4, the nanoparticles are almost spherical with acceptable uniformity. Trivial deviation from complete sphericity could be assigned to stresses exerted upon the nanospheres during congealing, for example, by the shear stress applied by the homogenizer or as a result of cross-linking. The SEM also confirms the particle size range already determined by zetasizer between 200 and $500 \mathrm{~nm}$.

3.4. Drug Loading Efficiency. The results of measuring of drug loading efficiency of different formulations are seen in Table 1 . As bupropion $\mathrm{HCl}$ is soluble in oily phase as well as in aqueous phase ( $\log K_{\mathrm{o} / \mathrm{w}}=3.21$ for bupropion), it is not unexpected to leave the agar solution during emulsification into 


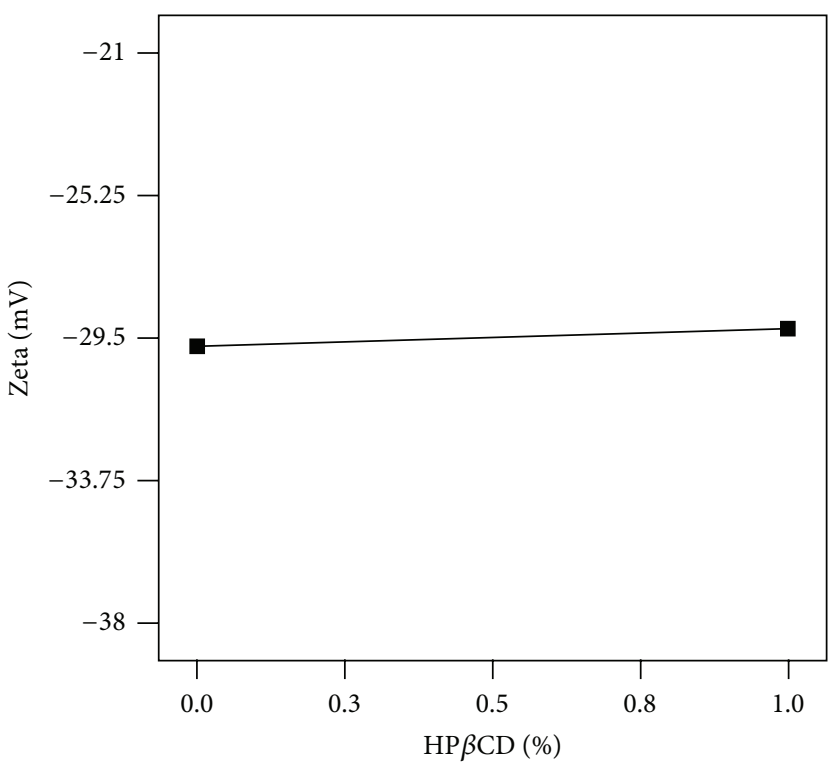

(a)

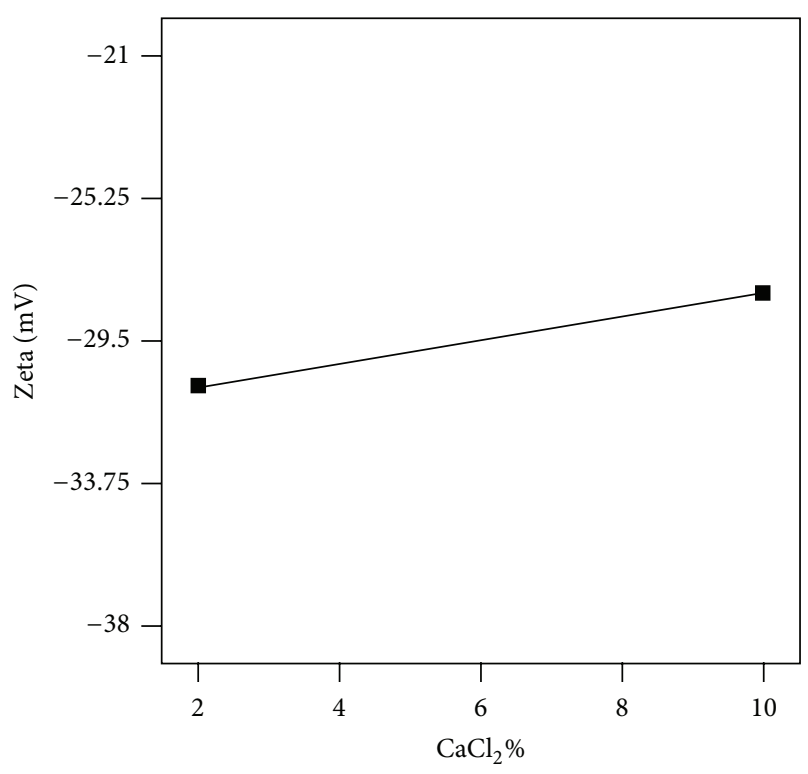

(b)

FIGURE 3: Correlation between zeta potential of agar nanospheres and (a) $\mathrm{HP} \beta \mathrm{CD} \%$ and (b) $\mathrm{CaCl}_{2} \%$.

the paraffin phase. Optimizing the time of homogenization has been tried to obtain acceptable load.

The results of Table 2 show that bupropion loading efficiency in the nanospheres was enhanced by increasing the agar content significantly $(P<0.05)$ while an antagonist effect was seen by the $\operatorname{HP} \beta C D(P<0.05)$.

As for the relationship between loading efficiency with input factors, calcium chloride caused more intimate entanglement of agar threads and constricted the pores through which bupropion molecules could not escape into the external phase during homogenizing. This in turn resulted in a higher loading efficiency (Figure 5). Higher speed of homogenizer by reducing the droplet size provided a larger surface for passing of the bupropion molecules into the oil phase and thus reduced the loading efficiency (Figure 5). HP $\beta C D$ probably played the role of a vehicle to transport the active molecules into the paraffin which again decreased the drug loading (Figure 5). Agar presented an agonist effect on the drug loading efficiency in the nanospheres (Table 2) which obviously meant that more agar could accommodate more drug in the nanospheres and this was the strongest or the most significant correlation between the input variables and the output or the responses (Figure 5).

3.5. In Vitro Drug Release Studies. Release efficiency after 300 minutes was calculated for all 25 formulations (Table 1). The minimum RE was $63 \%$ (for $\mathrm{C}_{6} \mathrm{~S}_{10000} \mathrm{~A}_{2} \mathrm{H}_{0.5}$ ) and the maximum was $91 \%$ (for $\mathrm{C}_{10} \mathrm{~S}_{12000} \mathrm{~A}_{2} \mathrm{H}_{1}$ ). Bupropion release profiles of all 25 formulations are shown in (Figures 6(a) to 6(d)).

The results of Table 2 show that $\mathrm{RE}_{300} \%$ of the drug was increased insignificantly $(P>0.05)$ by the $\mathrm{CaCl}_{2}$ percent and homogenization speed while it was decreased by the agar content $(P<0.05)$.

$\mathrm{RE}_{300}$ showed a mild increase with increasing amount of cross-linking agent (Figure 7) that can be explained by

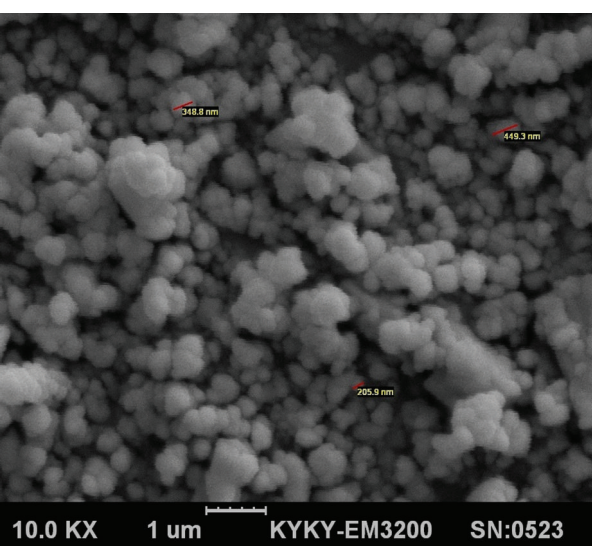

FIGURE 4: Scanning electron micrograph of agar nanospheres loaded with bupropion $\mathrm{HCl}$.

axial squeezing out of bupropion towards the periphery of the nanospheres as the result of inward contraction exerted by the cross-linker which in turn means increasing accumulation of bupropion in the peripheral area of the matrix of the nanospheres and more rapid release.

As Figure 7 shows, more contents of $\mathrm{HP} \beta C D$ caused more facilitated entrance of bupropion into the release medium which may be due to the solubilizing nature of $\mathrm{HP} \beta \mathrm{CD}$ causing greater $\mathrm{RE}_{300}$ (Figure 7).

3.6. Optimization. Numerical solution proposed by the software with the greatest desirability for the optimum formulation consisted of $7.19 \%$ calcium chloride, homogenization speed of $8500 \mathrm{rpm}, 2 \%$ of agar, and $0.12 \%$ of $\mathrm{HP} \beta \mathrm{CD}$. Response factors corresponding to these inputs, predicted by the software, should show particle size of $577.68 \mathrm{~nm}$, zeta 


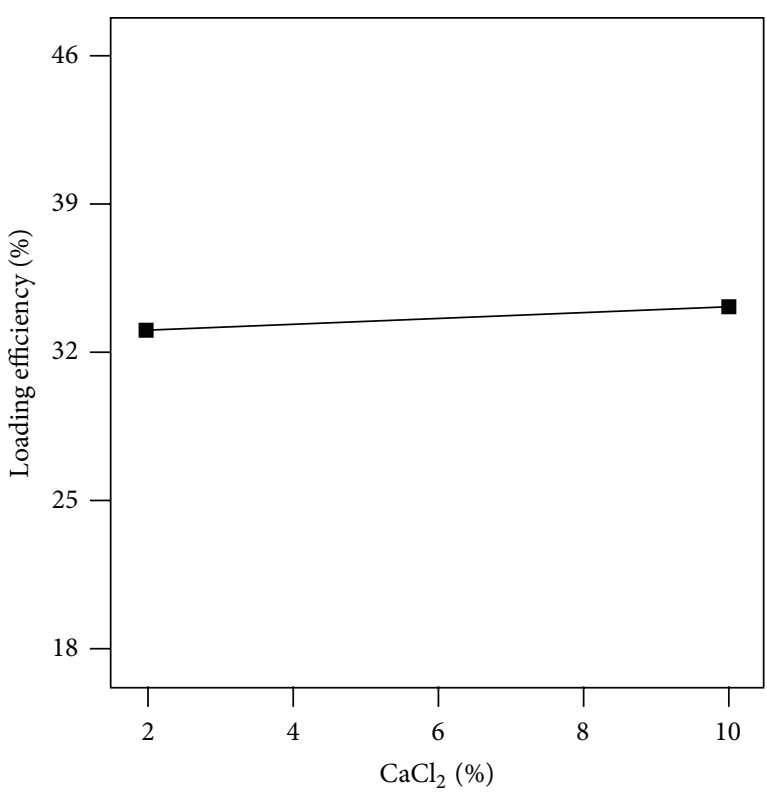

(a)

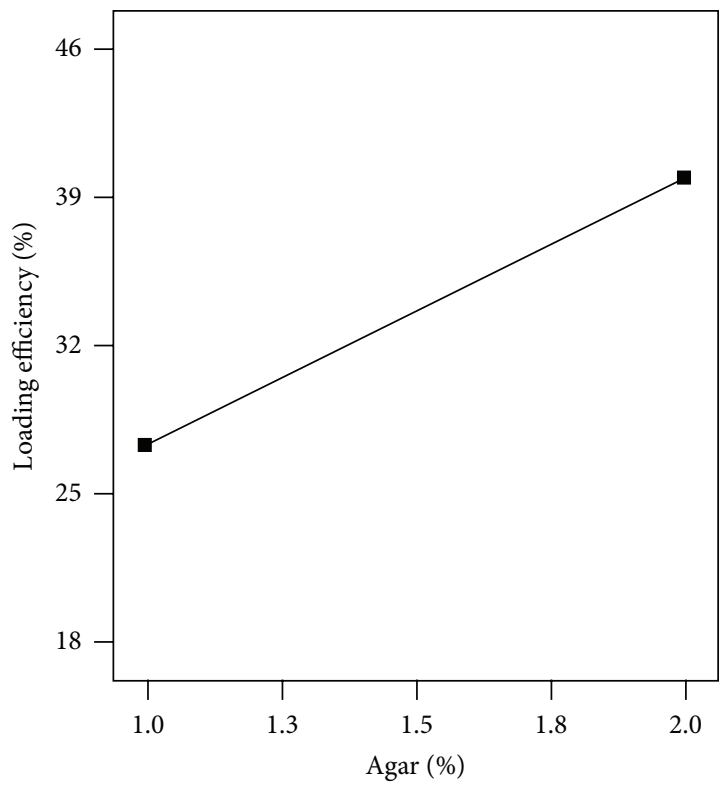

(c)

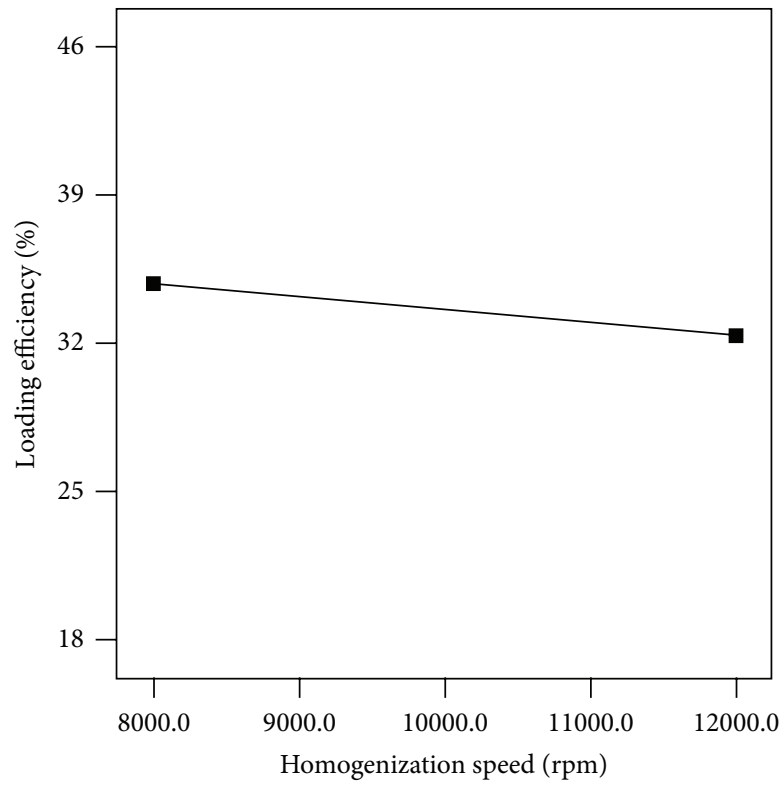

(b)

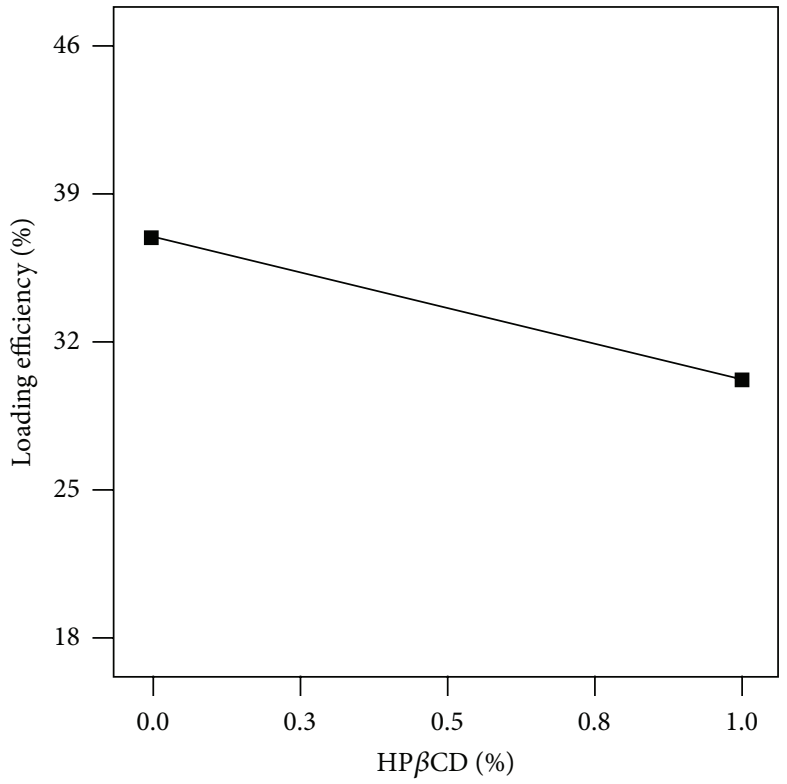

(d)

FigurE 5: Effect of (a) $\mathrm{CaCl}_{2} \%$, (b) homogenizer speed, (c) agar content, and (d) HP $\beta C D \%$ on drug loading efficiency in agar nanospheres.

potential of $-30 \mathrm{mV}$, loading efficiency of $43.5 \%$, and $\mathrm{RE}_{300}$ of $69.49 \%$. Comparison between the predicted and real values of these responses and their error percent is summarized in Table 4.

3.7. Mucoadhesion Evaluation. The optimized nanospheres were tested for mucoadhesion which showed that the adsorption of mucin solution on to the nanospheres was nearly complete (99.5\%). Therefore, it may be expected that these nanospheres will show strong mucoadhesion when applied to mucous and biomembranes of pulmonary route. Although the mucoadhesion test was carried out in acetate buffer due to the better dissolution of mucin, repeating of the test in simulated lung fluid (Gamble's Solution [16]) showed the mucoadhesion of $92.86 \%$ which was comparable to $99.5 \%$ obtained in acetate buffer. Some authors have reported that the airway surface liquid is slightly acidic, and this acidity might be part of normal airway defense. The $\mathrm{pH}$ value as low as 5.7 has been reported for the airway surface liquids by some authors [35]. Thus, $\mathrm{pH}$ of 4.5 in evaluating the mucoadhesion does not seem to adversely affect the results. Also, the presence of acetate ion in a majority of simulated lung fluids is mentioned in the literature [16].

As our preliminary studies showed, hydroxypropyl $\beta$ cyclodextrin did not show significant effect on the mucoadhesion of the nanospheres. However, to study its effect on 


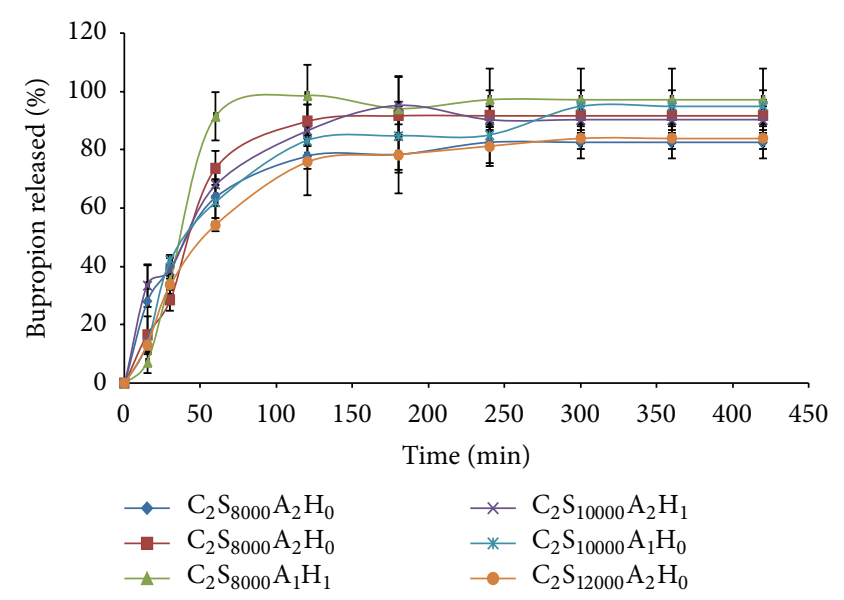

(a)

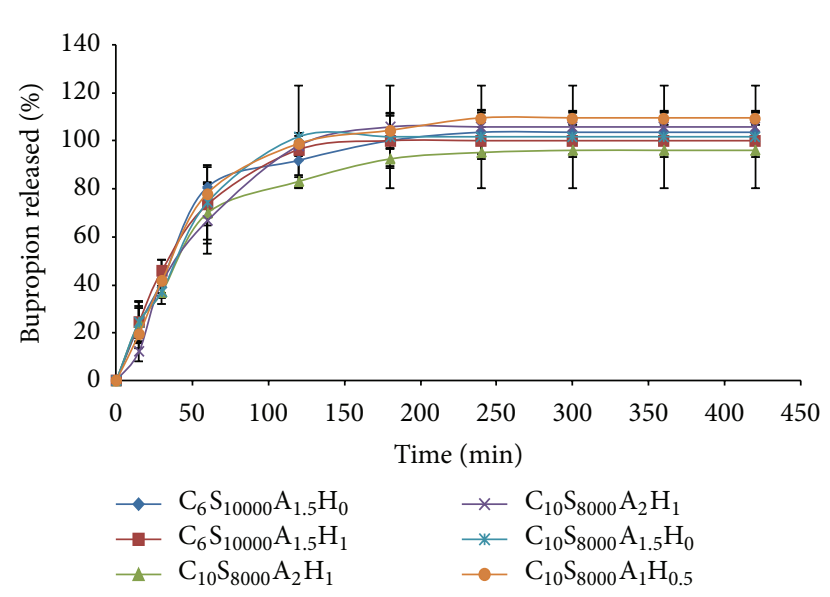

(c)

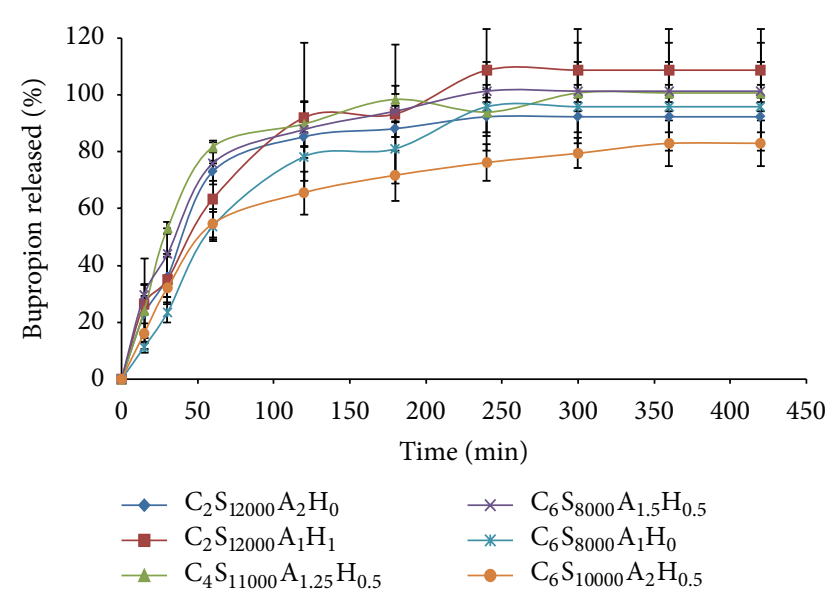

(b)

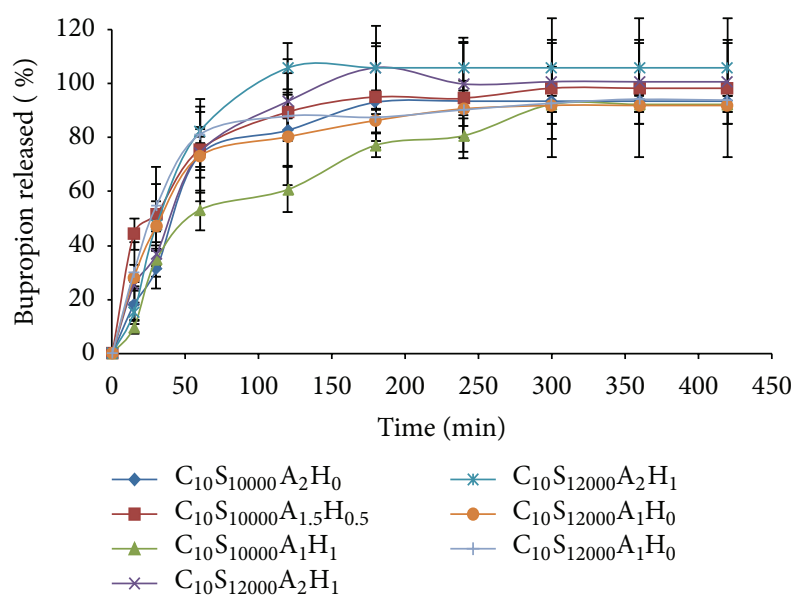

(d)

FIGURE 6: Release profiles of all 25 run, arranged based on the increasing amounts of $\mathrm{CaCl}_{2} \%$ and homogenizer speeds (the input factors with greater effects on release) from (a) to (d).

TABLE 4: Predicted versus real response factors for the optimum formulation (measured in deionized and purified water).

\begin{tabular}{lcccc}
\hline & Particle size $(\mathrm{nm})$ & Zeta potential $(\mathrm{mV})$ & Loading efficiency $(\%)$ & $\mathrm{RE}_{300}(\%)$ \\
\hline Predicted & 577.68 & -30.10 & 43.5 & 69.49 \\
Real & $587 \pm 58$ & -30.9 & $38.6 \pm 1.3$ & $51 \pm 9$ \\
Error percent & 1.61 & 2.65 & 11.26 & 26.6 \\
\hline
\end{tabular}

the mucoadhesion of the optimized formulation, it was prepared with various concentrations of hydroxypropyl $\beta$ cyclodextrin $(0,0.5$ and $1 \%)$ and the mucoadhesion of the optimized nanospheres was measured in acetate buffer. The results are shown in Table 5 . As this table indicates, the different concentrations of HP $\beta C D$ were not effective on the mucoadhesion of the nanospheres $(P>0.05)$.

3.8. Stability of the Nanospheres in the Simulated Lung Fluid. In the present work the nanospheres suspension is supposed to be administered into the rat lungs by using a microsprayer device which renders the suspension into microdroplets capable to reach the deepest parts of pulmonary tract. Therefore, the stability of the nanoparticles was studied in the simulated lung fluid during the time they reside there and release their drug content. The results of stability studies of nanospheres in simulated lung fluid (Gamble's solution [16]) are shown in Table 6. The absence of drug degradation or great increase of particle size and zeta potential of nanospheres shows its good stability during its life span and residence time in the lungs. Although the measurements of these parameters at zero time in the water and simulated lung fluid are different (Table 6), the changes after 9 hours in the simulated lung fluid does not show significant difference $(P>0.05)$. The absence of many size increments during the life span of the nanospheres in the simulated lungs' fluid along with their suitable zeta potential of $-11.6 \mathrm{mV}$ which is high enough to prevent the aggregation of the nanospheres 


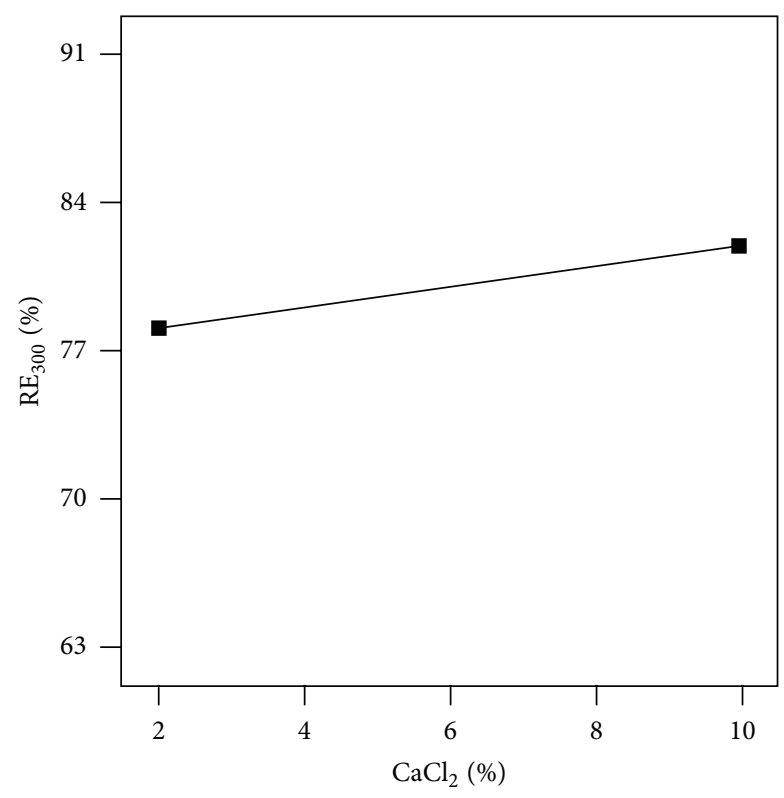

(a)

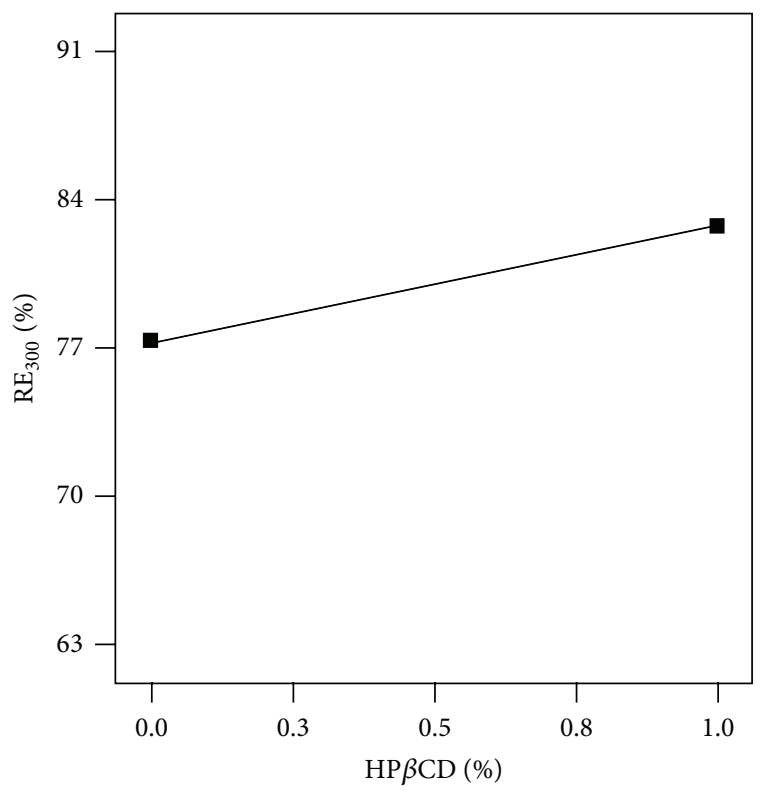

(b)

FIGURE 7: Variation of release efficiency of bupropion $\mathrm{HCl}$ from agar nanospheres with (a) $\mathrm{CaCl}_{2} \%$ and (b) $\mathrm{HP} \beta \mathrm{CD} \%$.

TABLE 5: The results of mucoadhesion measurement of the optimized agar nanospheres in different fluids and in different concentrations of hydroxypropyl $\beta$-cyclodextrin (in acetate buffer).

\begin{tabular}{lcccc}
\hline $\begin{array}{l}\text { Acetate } \\
\text { buffer }\end{array}$ & $\begin{array}{l}\text { Simulated } \\
\text { lung fluid }\end{array}$ & HP $\beta$ CD 0\% & $\begin{array}{c}\text { HP } \beta \text { CD } \\
0.5 \%\end{array}$ & HP $\beta$ CD 1\% \\
\hline $99.5 \%$ & $92.86 \%$ & $99.4 \%$ & $99.5 \%$ & $99.3 \%$ \\
\hline
\end{tabular}

TABLE 6: The results of measuring the particle size, zeta potential, and drug content of the optimum formulation measured in the simulated lung fluid [16] immediately and 9 hours after preparation.

\begin{tabular}{lccc}
\hline \multicolumn{2}{c}{ Particle size $(\mathrm{nm})$} & \multicolumn{2}{c}{ Zeta potential $(\mathrm{mV})$} \\
At zero time & After 9 hrs & At zero time & After 9 hrs \\
\hline $553 \pm 32$ & $600 \pm 40$ & -11.6 & -10.6 \\
\hline
\end{tabular}

guarantees their stability in the lung during the period of their drug release.

\section{Conclusions}

Loading of bupropion $\mathrm{HCl}$ into agar nanospheres was performed successfully. Along with the active ingredient, crosslinking agent and a permeability increasing agent (HP $\beta C D)$ were included in the nanospheres. Agar being a biodegradable/bioadhesive natural polymer is an attractive candidate for delivery of drugs in form of nanospheres via various biomembranes including pulmonary route. The relatively sufficient duration of release time (about 5 hours) as well as mucoadhesiveness of the nanospheres insures a considerable residence time of the nanospheres in vicinity of mucous membranes. The problem of stickiness of resulting agar nanospheres was overcome by freeze-drying or by simply adsorbing the nanospheres on mannitol powder. The optimum levels for studied independent factors proposed by the a D-optimal design were $7.19 \%$ of calcium chloride, homogenization speed of $8500 \mathrm{rpm}, 2 \%$ of agar, and $0.12 \%$ of $\mathrm{HP} \beta C D$. Response factors corresponding to these inputs, predicted by the software, were particle size of $577.68 \mathrm{~nm}$, zeta potential of $-30 \mathrm{mV}$, loading efficiency of $43.5 \%$, and $\mathrm{RE}_{300}$ of $69.49 \%$. Error percentages which give an estimate of the accordance between predicted and real values for the response factors were $1.61 \%$ and $2.65 \%$ for particle size and zeta potential, respectively, which are excellent. For loading efficiency, it was $11.26 \%$ which is rather acceptable but the error percent for $\mathrm{RE}_{300}$ is quiet large, that is, $26.6 \%$. However, the Doptimal design turns out to be a somewhat proper method to optimize the fabrication of agar nanospheres though with some limitations markedly apparent for release efficiency. Agar nanospheres also showed excellent bioadhesiveness in vitro. To demonstrate the effectiveness of the designed nanospheres in the pulmonary delivery of bupropion, more detailed studies are performing on animal model in vivo.

\section{Conflict of Interests}

The authors declare that there is no conflict of interests regarding the publication of this paper.

\section{Acknowledgment}

The authors acknowledge the financial support of Isfahan University of Medical Sciences.

\section{References}

[1] S. Casey Laizure, C. Lindsay DeVane, J. T. Stewart, C. S. Dommisse, and A. A. Lai, "Pharmacokinetics of bupropion and 
its major basic metabolites in normal subjects after a single dose," Clinical Pharmacology and Therapeutics, vol. 38, no. 5, pp. 586-589, 1985.

[2] L. L. Brunton, J. S. Lazo, and K. L. Parker, Eds., Goodman \& Gilman's the Pharmacological Basis of Therapeutics, McGrawHill, New York, NY, USA, 2006.

[3] S. M. Stahl, J. F. Pradko, B. R. Haight, J. G. Modell, C. B. Rockett, and S. L. Coughlin, "A review of the neuropharmacology of bupropion, a dual norepinephrine and dopamine reuptake inhibitor," The Primary Care Companion-Journal of Clinical Psychiatry, vol. 6, no. 4, pp. 159-166, 2004.

[4] N. B. Mehta, "Meta-chloro-substituted alpha-butylaminopropiophenones," US Patent 3,819,706, 1974.

[5] R. A. Sweet, B. G. Pollock, M. Kirshner, B. Wright, L. P. Altieri, and C. L. DeVane, "Pharmacokinetics of single- and multipledose bupropion in elderly patients with depression," Journal of Clinical Pharmacology, vol. 35, no. 9, pp. 876-884, 1995.

[6] R. F. Suckow, T. M. Smith, A. S. Perumal, and T. B. Cooper, "Pharmacokinetics of bupropion and metabolites in plasma and brain of rats, mice, and guinea pigs," Drug Metabolism and Disposition, vol. 14, no. 6, pp. 692-697, 1986.

[7] W. W. K. Zung, H. K. H. Brodie, L. Fabre, D. McLendon, and D. Garver, "Comparative efficacy and safety of bupropion and placebo in the treatment of depression," Psychopharmacology, vol. 79, no. 4, pp. 343-347, 1983.

[8] D. E. Jorenby, J. T. Hays, N. A. Rigotti et al., "Efficacy of varenicline, an $\alpha 4 \beta 2$ nicotinic acetylcholine receptor partial agonist, vs placebo or sustained-release bupropion for smoking cessation: a randomized controlled trial," Journal of the American Medical Association, vol. 296, no. 1, pp. 56-63, 2006.

[9] L. A. Labbate, J. B. Grimes, A. Hines, and M. H. Pollack, "Bupropion treatment of serotonin reuptake antidepressantassociated sexual dysfunction," Annals of Clinical Psychiatry, vol. 9, no. 4, pp. 241-245, 1997.

[10] R. A. Plodkowski, Q. Nguyen, U. Sundaram, L. Nguyen, D. L. Chau, and S. St Jeor, "Bupropion and naltrexone: a review of their use individually and in combination for the treatment of obesity," Expert Opinion on Pharmacotherapy, vol. 10, no. 6, pp. 1069-1081, 2009.

[11] D. P. Cantwell, "ADHD through the life span: the role of bupropion in treatment," The Journal of Clinical Psychiatry, vol. 59, supplement 4, pp. 92-94, 1997.

[12] J. G. Modell, N. E. Rosenthal, A. E. Harriett et al., "Seasonal affective disorder and its prevention by anticipatory treatment with bupropion XL," Biological Psychiatry, vol. 58, no. 8, pp. 658667, 2005.

[13] A. L. Brody, A. G. Mukhin, M. S. Mamoun et al., "Treatment for tobacco dependence: effect on brain nicotinic acetylcholine receptor density," Neuropsychopharmacology, vol. 38, no. 8, pp. 1548-1556, 2013.

[14] D. Zhang, B. Yuan, M. Qiao, and F. Li, "HPLC determination and pharmacokinetics of sustained-release bupropion tablets in dogs," Journal of Pharmaceutical and Biomedical Analysis, vol. 33, no. 2, pp. 287-293, 2003.

[15] P. H. Silverstone, R. Williams, L. McMahon, R. Fleming, and S. Fogarty, "Convulsive liability of bupropion hydrochloride metabolites in Swiss albino mice," Annals of General Psychiatry, vol. 7, article 19, 2008.

[16] M. R. Marques, R. Loebenberg, and M. Almukainzi, "Simulated biological fluids with possible application in dissolution testing," Dissolution Technologies, vol. 18, no. 3, pp. 15-28, 2011.
[17] N. Wang and X. S. Wu, "Preparation and characterization of agarose hydrogel nanoparticles for protein and peptide drug delivery," Pharmaceutical Development and Technology, vol. 2, no. 2, pp. 135-142, 1997.

[18] L. E. Ju, J. K. Park, S. A. Khan, and K. H. Lim, "Preparation of agar nanoparticles by W/O emulsification," Journal of Chemical Engineering of Japan, vol. 44, pp. 502-508, 2011.

[19] N. Wang and X. S. Wu, "A novel approach to stabilization of protein drugs in poly(lactic-co-glycolic acid) microspheres using agarose hydrogel," International Journal of Pharmaceutics, vol. 166, no. 1, pp. 1-14, 1998.

[20] K. Bera, K. K. Sarwa, and B. Mazumder, "Metformin HCl loaded mucoadhesive agar microspheres for sustained release," Asian Journal of Pharmaceutics, vol. 7, no. 2, pp. 75-82, 2013.

[21] U. Bilati, E. Allémann, and E. Doelker, "Development of a nanoprecipitation method intended for the entrapment of hydrophilic drugs into nanoparticles," European Journal of Pharmaceutical Sciences, vol. 24, no. 1, pp. 67-75, 2005.

[22] H. H. Selby and W. H. Wynne, "Agar," in Industrial Gums, R. L. Whistler, Ed., pp. 29-49, Academic Press, New York, NY, USA, 1973.

[23] K. Manjunatha, M. Ramana, and D. Satyanarayana, "Design and evaluation of diclofenac sodium controlled drug delivery systems," Indian Journal of Pharmaceutical Sciences, vol. 69, no. 3, pp. 384-389, 2007.

[24] S. Hjertén, “The preparation of agarose spheres for chromatography of molecules and particles," Biochimica et Biophysica Acta-Specialized Section on Biophysical Subjects, vol. 79, no. 2, pp. 393-398, 1964.

[25] Y. Mu, A. Lyddiatt, and A. W. Pacek, "Manufacture by water/oil emulsification of porous agarose beads: effect of processing conditions on mean particle size, size distribution and mechanical properties," Chemical Engineering and Processing: Process Intensification, vol. 44, no. 10, pp. 1157-1166, 2005.

[26] A. M. Egorov, A. K. Vakhabov, and V. Y. Chernyak, "Isolation of agarose and granulation of agar and agarose gel," Journal of Chromatography A, vol. 46, pp. 143-148, 1970.

[27] Z. Linghui, Y. Ling-ling, Z. Ling-min, J. Xing-long, T. XunQing, and C. Ji-Ye, 2011, http://www.paper.edu.cn/.

[28] F. Tewes, O. L. Gobbo, M. I. Amaro et al., "Evaluation of HP $\beta$ CD-PEG microparticles for salmon calcitonin administration via pulmonary delivery," Molecular Pharmaceutics, vol. 8, no. 5, pp. 1887-1898, 2011.

[29] K. K. Loboz, A. S. Gross, J. Ray, and A. J. McLachlan, "HPLC assay for bupropion and its major metabolites in human plasma," Journal of Chromatography B: Analytical Technologies in the Biomedical and Life Sciences, vol. 823, no. 2, pp. 115-121, 2005.

[30] J. Varshosaz and Z. Dehghan, "Development and characterization of buccoadhesive nifedipine tablets," European Journal of Pharmaceutics and Biopharmaceutics, vol. 54, no. 2, pp. 135-141, 2002.

[31] Y.-F. Maa and C. Hsu, "Liquid-liquid emulsification by rotor/stator homogenization," Journal of Controlled Release, vol. 38, no. 2-3, pp. 219-228, 1996.

[32] S. Tcholakova, N. D. Denkov, D. Sidzhakova, I. B. Ivanov, and B. Campbell, "Interrelation between drop size and protein adsorption at various emulsification conditions," Langmuir, vol. 19, no. 14, pp. 5640-5649, 2003. 
[33] G. Narsimhan and P. Goel, "Drop coalescence during emulsion formation in a high-pressure homogenizer for tetradecane-inwater emulsion stabilized by sodium dodecyl sulfate," Journal of Colloid and Interface Science, vol. 238, no. 2, pp. 420-432, 2001.

[34] G. Narsimhan, "Model for drop coalescence in a locally isotropic turbulent flow field," Journal of Colloid and Interface Science, vol. 272, no. 1, pp. 197-209, 2004.

[35] H. Fischer and J. H. Widdicombe, "Mechanisms of acid and base secretion by the airway epithelium," Journal of Membrane Biology, vol. 211, no. 3, pp. 139-150, 2006. 

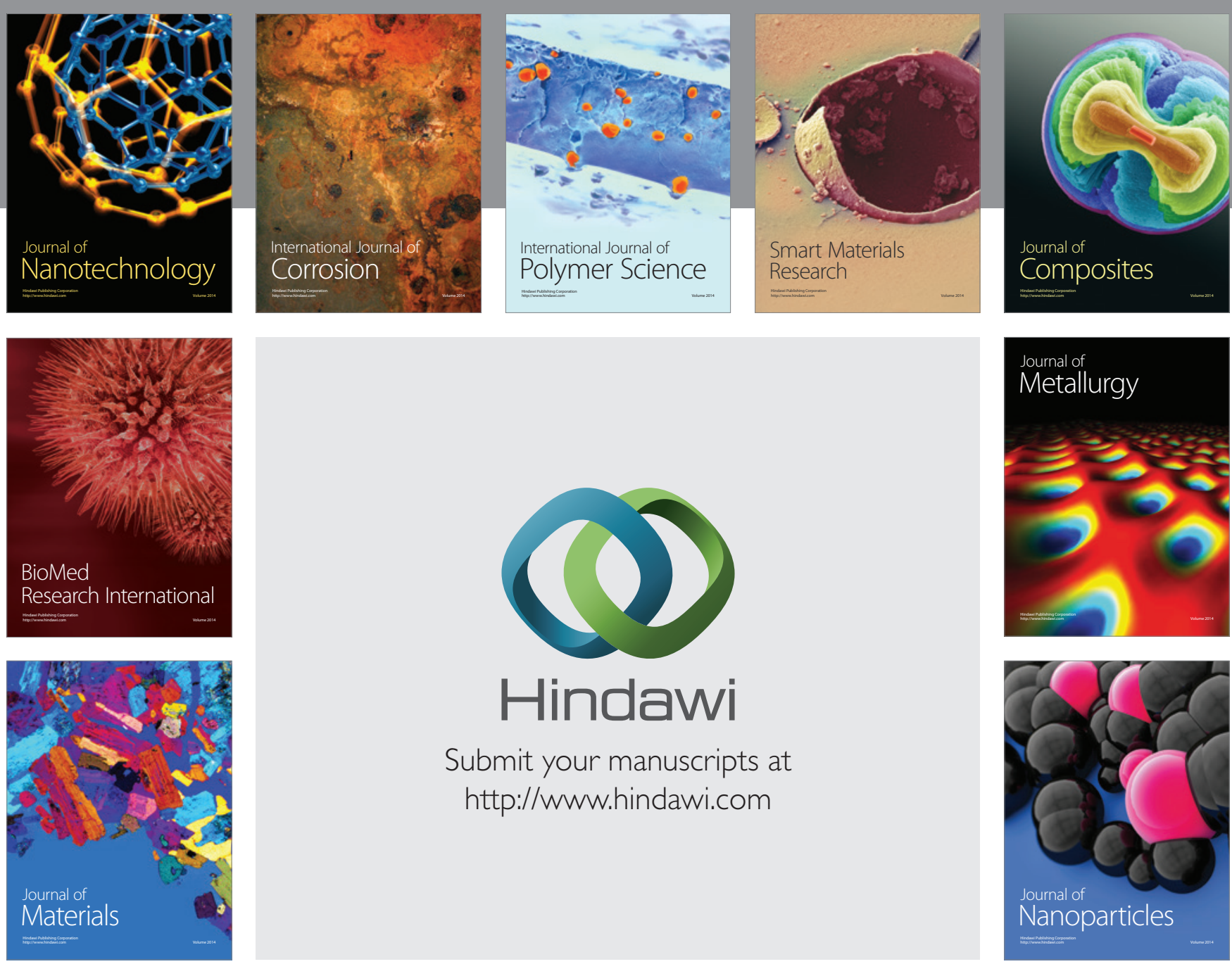

Submit your manuscripts at http://www.hindawi.com
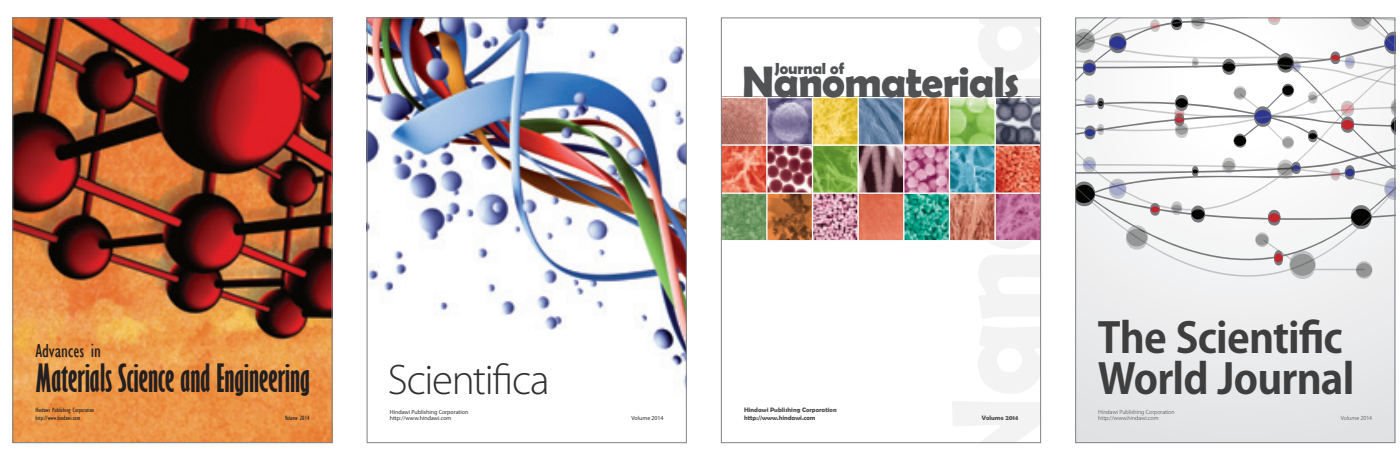

\section{The Scientific World Journal}
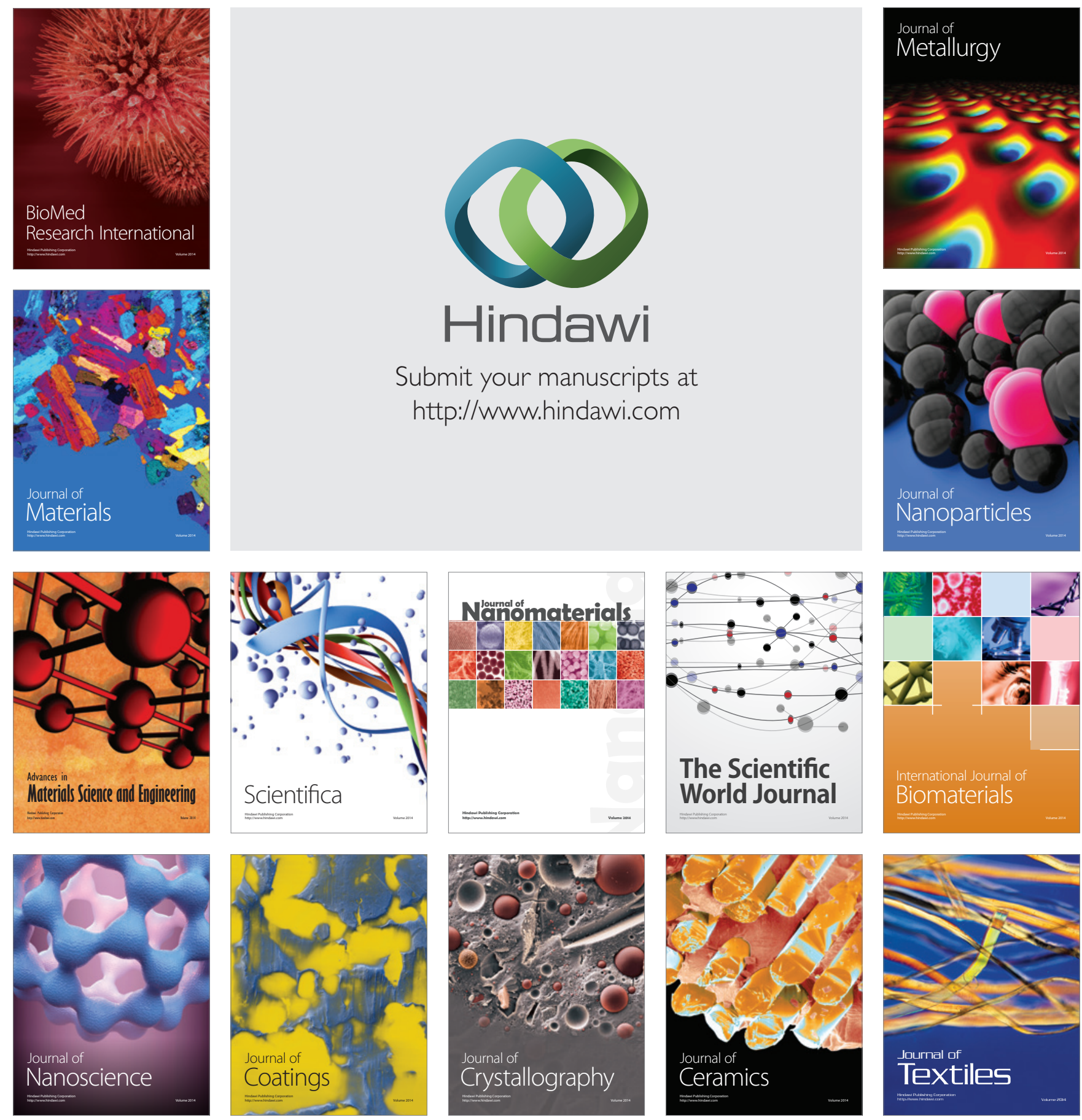